\author{
Dan A. Svensson \\ Elisabet Waldenlind \\ Karl Ekbom \\ Nancy L. Pedersen
}

\section{Heritability of migraine as a function of definition}

D. Svensson ( $₫)$

Neurotec Department,

Division of Neurology,

Karolinska Institutet,

Karolinska University Hospital,

Huddinge, R-54,

S-14186 Stockholm, Sweden

e-mail: Dan.Svensson@neurotec.ki.se

Tel.: +46-8-5858-2258

Fax: +46-8-774-4822

E. Waldenlind • K. Ekbom

Department of Neurology,

Karolinska Institutet,

Karolinska University Hospital,

Huddinge, Stockholm, Sweden

N. Pedersen

Department of Epidemiology and

Biostatistics,

Karolinska Institutet,

Stockholm, Sweden

\begin{abstract}
The objective was to examine the main genetic and environmental architecture of migraine in the Screening Across Lifetime of Twin (SALT) Study as a function of definition. We performed a quantitative genetic study of the Swedish population and a total of 12,095 twin pairs aged $41-64$ years were interviewed on the telephone by trained lay personnel using a structured questionnaire. Lifetime assessment of recurrent headache and two measures of migraine were obtained, diagnosis in line with the 1988 International Headache Society criteria and self-report. The lifetime prevalence was $10.3 \%$ for diagnosed and $21.4 \%$ for selfreported migraine. Among diagnosed cases $82.3 \%$ self-reported migraine. Among self-ascertained cases, $60.3 \%$ did not fulfil diagnostic criteria, and one third had not
\end{abstract}

had recurrent headache. In multivariate analyses, genetic influences were common to diagnosed and self-reported migraine (42\%) and specific to self-report migraine $(11 \%)$. In univariate analyses, heritability was stable (52\%) when "false positives" were removed from the case definition (diagnosed or self-reported migraine) and decreased the prevalence from 23 to $16 \%$ but dropped (from 52 to $38 \%$ ) when "false negatives" were removed and decreased the prevalence from 23 to $17 \%$. A relationship between the definition and the heritability of migraine was demonstrated and phenotypes of clinical and aetiologic relevance were disclosed.

Key words Migraine $\cdot$ Genetics • Heritability • Twin study

\section{Introduction}

Migraine appears to be a complex disorder caused by influences of multiple genes and environmental factors [1]. A number of migraine genes have been implicated for rare autosomal dominant traits and other familial cases [2]. These genes may contribute to a better understanding of disease mechanisms. However, their role is unclear for occurrence of migraine in the general population, where health care utilisation is limited and the threshold somewhat arbitrary.
The importance of genes for migraine has been indicated by observations of a relatively larger proportion of concordant monozygous than dizygous twin pairs [3-8]. In large twin cohorts of the population-based Scandinavian twin registries, the relative importance of genetic influences on liability to migraine (i.e., heritability) has been estimated to be $50-60 \%$ [7-10]. A genetic effect for migraine has been confirmed using the adopted twin design [11, 12]. It seems that migraine manifestation during childhood and adolescence is mainly due to genetic effects, whereas migraine manifestation during adulthood 
involves the individual specific environment to a larger extent $[13,14]$. Diagnostic criteria and data collection methods influence the threshold of migraine [15-17]. A relationship between the definition of disease and the heritability may provide guidance as to how to delineate phenotypes of clinical and aetiologic relevance.

The purpose of the present study was to examine the main genetic and environmental architecture of migraine in a large population-based cohort of twin pairs using varying definitions of illness as defined by clinical consensus criteria and self-report. A screening for common, complex disorders was performed in the Swedish Twin Registry through computer assisted telephone interviews and all twins aged 41-64 years were surveyed for migraine.

\section{Subjects and methods}

\section{Study population}

All twins born 1935-1946 ( $\mathrm{n}=46,286$ twins) were recruited from a nationwide register of Swedish twins [18]. As part of the Screening Across the Lifespan of Twins (SALT) study, twins were invited to participate in a structured telephone interview concerning common, complex disorders including headaches. Trained lay personnel conducted interviews between 1 March 1999 and 22 December 2002. Vital status, family name and address were updated monthly with a national registry and telephone numbers were obtained by linkage to the Swedish national telephone catalogue. During each month of the data collection period a random sample of about 1000 twins was drawn, and introductory letters were mailed to these twins. In total, 41,000 twins were invited to participate in the SALT Headache study. Reasons for not inviting twins were death, serious disease, residence abroad and missing address and/or telephone number. A total of 31,192 twins (76\%) participated in the SALT Headache study. After exclusion of twins with incomplete information and twins representing broken pairs, 12,095 twin pairs were eligible for further analyses. The mean age of these twins was 52.0 years ( $\mathrm{SD}=6.5$ years). Out of all eligible pairs, $37 \%$ were unlike-sexed dizygous (DZ) pairs. Out of the like-sexed pairs, $55 \%$ were female and $42 \%$ monozygous (MZ) pairs. For analytical purposes, twin pairs were split into five groups: male/male MZ ( $\mathrm{n}=1419)$, male/male DZ $(\mathrm{n}=2019)$, female/female MZ ( $\mathrm{n}=1802)$, female/female DZ $(\mathrm{n}=2398)$, and male/female DZ $(n=4457)$. The Swedish Data Inspection authority and the local Ethics Committee gave sanction to the study.

\section{Migraine assessment}

An interview questionnaire consisted of short questions in regard to migraine symptoms. A lifetime history of recurrent headache not associated with infection, fever or hangover worked as a mandatory gating criterion for primary headache. Migraine was defined in accordance to the 1988 International Headache Society (IHS) criteria for migraine without aura [19], where the frequen- cy criterion of at least 5 attacks was met by the presence of recurrent headache. In addition, a few respondents who declared that they did not know whether they had had headache attacks lasting from 4 hours to 3 days, but who fulfilled all other criteria, were also diagnosed as sufferers of migraine ( $n=136$ twins). As an independent marker of migraine, all twins were asked whether they had ever suffered from migraine [16, 20, 21]. Varying definitions of migraine were obtained using diagnosed and selfreported measures of migraine as shown in Table 1.

Quantitative genetic analyses

Quantitative genetic modelling of binary migraine data in the five groups of twin pairs was performed as described in detail by Neale and Cardon [22]. To estimate the relative importance of genetic and environmental influences, a path-modelling framework of the classical twin model as implemented in the Mx structural equation modelling software was used [23]. Standard Mx scripts were used to carry out the analyses. The co-variance of IHS-migraine and self-report migraine was partitioned into genetic and environmental components using a multivariate model, i.e., the phenotypic Cholesky decomposition model. The extent to which the same genetic and environmental effects contribute to co-variation of IHS-migraine and self-report migraine was computed. Liability of definitions of migraine that were derived was decomposed into genetic and environmental influences using univariate models.

In the basic path model for twin designs, latent additive (A) and non-additive (D) genetic factors contribute to phenotypic similarity within pairs, where the expected twin correlation is 1.0 for $\mathrm{A}$ and $\mathrm{D}$ in $\mathrm{MZ}$ twins and 0.5 for $\mathrm{A}$ and 0.25 for $\mathrm{D}$ in $\mathrm{DZ}$ twins. In the classical twin design of twins reared together from birth to adulthood, a shared family environment (C) factor may replace $\mathrm{D}$, where the expected twin correlation is assumed to be 1.0 in both $\mathrm{MZ}$ and $\mathrm{DZ}$ twins. An individual specific environment (E) factor represents residual variance that makes family members different from each other. The effect of latent factors on variability of phenotype is estimated as path coefficients of known

Table 1 Definitions of migraine

\section{Criteria}

(1) IHS $1.1^{\dagger}$

(2) Self-report migraine

(3) Other recurrent headache

(4) No other recurrent headache

Definitions

Strict migraine: (1)

Broad migraine: (1) or (2) and (3)

Broad' migraine: (1) or (2) and (4)

Super-broad migraine: (1) or (2)

$\dagger$ Recurrent headache not associated with infection, fever or hangover (past or present) \& attacks lasting 4-72 h \& at least two out of four pain characteristics (moderate or severe intensity, unilateral location, pulsation quality and aggravation by physical activity) $\&$ at least one of three accompanying symptoms (nausea, vomiting and increased sensitivity to light and sound) 
statistical properties. These parameter estimates are expressed in terms of proportions of the total phenotypic variance, where genetic effects provide estimates of the heritability. As a rule of thumb, an additive genetic effect is anticipated if phenotypic within-pair similarity of MZ twins is twice that of DZ twins, whereas a larger difference indicates a non-additive genetic effect and a smaller difference a shared environmental effect. Sex-specific effects (different genetic or environmental influence across the sexes) are indicated by a lower phenotypic within-pair similarity in unlike-sexed DZ twins than in like-sexed DZ twins.

The various definitions of migraine were assumed to reflect an underlying normal distribution of liability, where a threshold value divides the probability distribution into the proportions of affected and non-affected subjects (i.e., a normal transformed prevalence). The tetra-choric correlation coefficient measures the association of liability between two binary variables. Dichotomous data of twin pairs summarised into a $2 \times 2$ contingency table have an underlying bivariate distribution that is defined by two thresholds, one for each twin, and the tetra-choric correlation (twin correlation). The tetra-choric correlation of one binary variable in twin 1 and another binary variable in twin 2 (cross-trait correlation) as well as two binary variables in one of the twins (self correlation) may be evaluated.

We first computed tetra-choric correlation coefficients of IHSmigraine and self-report migraine in a pair-wise fashion to generate matrices of correlations (twin, cross-trait and self correlations) for each of the sex by zygosity groups using the software PRELIS [24]. Variance-covariance matrices of correlations and asymptotic weight matrices were read into the appropriate multivariate $\mathrm{Mx}$ script. Next, univariate models were fitted to the sex by zygosity $2 \times 2$ contingency tables for each of the definitions of migraine that were derived. The thresholds of the univariate models were allowed to differ between the sexes. The total variance was constrained to be 1, i.e., a standardised liability-threshold model. Models without and with sex-differences of variance components were fitted. Sex-specific effects were analysed using unconstrained correlations of the latent factors for unlike-sexed DZ twins.

Full models were fitted to the data (ADE or ACE models). Significance of model factor(s) was assessed by means of the difference in chi-square goodness of fit between the full model and a nested sub-model, where the factor(s) of interest is dropped. The chi-square difference test is chi-square distributed with degrees of freedom equal to the difference in number of unique parameters between full and restricted models. If twin correlations did not indicate shared environmental influences, results are shown for ADE-models, and if twin correlations did not indicate sex-limited effects, results of such analyses are not shown. Mx provided the likelihood-based $95 \%$ confidence interval and model fit statistics including Aikaike's Information Criterion ( $\mathrm{AIC}=\chi^{2}-2$ degrees of freedom). A model representing the best combination of fit and parsimony has the lowest value on AIC.

\section{Results}

Out of 24,190 twins, 6244 had ever had recurrent headache (25.8\%). Among 2501 headache sufferers with migraine according to the IHS-diagnostic instrument, all with the exception of 443 cases self-reported migraine (82.3\%). Among 5201 twins with self-report migraine 2058 had IHS-migraine $(39.6 \%)$. The lifetime prevalence of recurrent headache by self-report migraine is shown in Table 2. The migraine criteria that were not fulfilled among respondents with self-report migraine and other recurrent headache included the duration criterion $(71 \%)$, the pain characteristics $(16 \%)$ and the associated symptoms (13\%).

The self-correlation of IHS-migraine and self-report migraine was large and did not differ by zygosity $(r \geq 0.7)$. Twin and cross-trait correlations were of similar magnitude. All within-pair correlations were larger in the MZ twins $(r$; range $=0.25-0.55)$ than in the DZ twins $(r$; range $=0.04-0.24)$.

Table 2 Recurrent headache by self-report migraine: lifetime prevalence $(\%)$

\begin{tabular}{lrrrr}
\hline & \multicolumn{4}{c}{ Self-report migraine } \\
\cline { 2 - 5 } & \multicolumn{2}{c}{ With (n) } & Without (n) \\
\hline IHS-migraine & 8.5 & $(2058)$ & 1.8 & $(443)$ \\
Other recurrent headache & 5.9 & $(1438)$ & 9.5 & $(2305)$ \\
No other recurrent headache & 7.0 & $(1705)$ & 67.1 & $(16,241)$ \\
\hline
\end{tabular}

Table 3 Migraine by varying definitions of illness: lifetime prevalence and heritability

\begin{tabular}{|c|c|c|c|c|c|c|c|}
\hline & \multicolumn{2}{|c|}{ Lifetime prevalence } & \multirow{2}{*}{$\frac{\text { Heritability } \dagger}{\%(95 \% \mathrm{CI})}$} & \multicolumn{4}{|c|}{ Model fit } \\
\hline & $\%(95 \% \mathrm{CI})$ & $\mathrm{F}: \mathrm{M}(95 \% \mathrm{CI}) \ddagger$ & & $\chi^{2}$ & $p$ & AIC & df \\
\hline Strict migraine & $10.3(9.8,10.6)$ & $3.8(3.5,4.2)$ & $(36,52)^{* *}$ & 14.985 & 0.242 & -9.015 & 12 \\
\hline Broad migraine & $16.3(15.8,16.8)$ & $2.9(2.7,3.1)$ & $(46,57)^{* *}$ & 4.559 & 0.971 & -19.441 & 12 \\
\hline Broad' migraine & $17.4(16.8,17.8)$ & $2.8(2.6,3.0)$ & $(31,44)^{*}$ & 12.387 & 0.415 & -11.613 & 12 \\
\hline Super-broad migraine & $23.3(22.8,23.8)$ & $2.6(2.4,2.8)$ & $(47,57)^{* *}$ & 11.736 & 0.467 & -12.264 & 12 \\
\hline
\end{tabular}

AIC, Akaike's Informative Criterion; $d f$, degrees of freedom

$\dagger$ Additive and non-additive genetic variance (ADE-model)

$\$$ The female-to-male ratio as evaluated by the logistic regression model and computation of odds-ratio

*The A-factor (additive genetic variance) is significant as evaluated by means of the chi-square difference test $(p<0.05)$

$* *$ The D-factor (non-additive genetic variance) is significant as evaluated by means of the chi-square difference test $(p<0.05)$ 
Using quantitative genetic modelling of the two measures of migraine, the heritability of liability was $43 \%$ according to IHS-based diagnosis and 53\% according to self-report $\left(\chi^{2}=33.967, p=0.968, \mathrm{df}=51\right)$. Genetic influences for selfreport migraine were in common with diagnosed migraine $(42 \%)$ and specific $(11 \%)$. The heritability range for various definitions was from $38 \%$ to $52 \%$ (Table 3 ). The magnitude of genetic and environmental influences did not differ between the sexes (Tables 3 and 4).

\section{Discussion}

In this work we replicate previous findings of a major genetic effect for migraine [7-14]. The main genetic and environmental architecture of migraine appears to be remarkably stable across Swedish birth cohorts (Table 5). In addition, the proportion of genetic variance due to non-additive genetic effects for lifetime migraine was almost identical for twins born 1886-1925 and twins born 1935-1958 (65\% vs.
$86 \%$ ). It is important and interesting to note that environmental changes over time have not modified the heritability of migraine although dramatic changes of circumstances and habits by which people live their life have occurred during the 20th century. However, it may be possible that factors of changing life circumstances and habits influence aspects of the migraine disease, such as the prognosis, the frequency of attacks and chronic development.

We employed two measures of migraine, an IHS-based diagnosis and self-report. The majority of diagnosed cases self-reported migraine and genetic correlation of the two variables was high. It seems thus that self-awareness of migraine is high in the Swedish population. However, many subjects self-reported migraine but did not fulfil diagnostic criteria. In addition, specific genetic effects were found for self-report migraine although of rather small magnitude. Analyses of varying definitions of disease revealed interesting features of the phenotype. First, starting from the most liberal definition of migraine (as measured by diagnosis or self-report), heritability was stable $(52 \%)$ when cases of self-ascertained migraine without other recurrent headache was considered to

Table 4 Variance components (\%) for migraine by various definitions of illness and sex with $95 \%$ confidence intervals between parentheses

\begin{tabular}{|c|c|c|c|c|c|c|c|c|c|c|c|}
\hline & \multicolumn{3}{|c|}{ Men } & \multicolumn{4}{|c|}{ Women } & \multicolumn{4}{|c|}{ Model fit } \\
\hline & $\mathrm{A}$ & $\mathrm{D}$ & $\mathrm{E}$ & A & $\mathrm{D}$ & & $\mathrm{E}$ & $\chi^{2}$ & $p$ & AIC & df \\
\hline Strict migraine & $0(0,45)$ & $33(0,51)$ & $67(49,88)$ & $6(0,32)$ & $40(10,54)^{*}$ & 54 & $(46,62)$ & 13.175 & 0.155 & -4.825 & 9 \\
\hline Broad migraine & $38(0,57)$ & $10(0,57)$ & $52(41,64)$ & $1(0,23)$ & $52(27,60)^{*}$ & 47 & $(40,54)$ & 2.582 & 0.979 & -15.418 & 9 \\
\hline Broad' migraine & $29(0,41)$ & $2(0,42)$ & $70(57,82)$ & $18(0,39)$ & $(0,46)$ & 60 & $(52,67)$ & 10.567 & 0.307 & -7.433 & 9 \\
\hline Super-broad migraine & $44(3,54)^{*}$ & $1(0,45)$ & $55(45,64)$ & $6(0,24)$ & $49(28,61)^{*}$ & 45 & $(39,51)$ & 7.244 & 0.612 & -10.756 & 9 \\
\hline
\end{tabular}

$A$, Additive genetic variance; $D$, Non-additive genetic variance; $E$, Individual specific environment variance; $A I C$, Akaike's Informative Criterion; $d f$, degrees of freedom

*The model-factor is significant as evaluated by means of the chi-square difference test $(p<0.05)$

Table 5 Heritability $\left(\mathrm{h}^{2}\right)$ of migraine in three classical twin studies $\dagger$

\begin{tabular}{lrrlllll}
\hline Birth cohorts & $\mathrm{n}$ (pairs) & Age & Time period & Migraine assessment & Prevalence, $\%$ & $\mathrm{~F}: \mathrm{M} \neq$ & $\mathrm{h}^{2}, \%$ \\
\hline $1935-1958^{1}$ & 12,095 & $41-64$ & Lifetime & $\begin{array}{l}\text { Recurrent headache \& IHS } \\
\text { or self-report migraine (CATI) }\end{array}$ & 16.3 & $2.9: 1$ & $52(\mathrm{D} *)$ \\
$1886-1925^{2,3}$ & 6080 & $42-81$ & Lifetime & $\begin{array}{l}\text { Self-report migraine } \\
\text { \& migraine symptoms (Q) }\end{array}$ & 20.4 & $2.5: 1$ & $55(\mathrm{D} *)$ \\
$1926-1958^{2,4}$ & 12,606 & $15-47$ & Recent years & $\begin{array}{l}\text { Severe disabling recurrent headache } \\
\text { \& migraine symptoms (Q) }\end{array}$ & 4.6 & $2.9: 1$ & $51($ Age*) \\
\hline
\end{tabular}

$I H S$, International Headache Society criteria; $C A T I$, Computer Assisted Telephone Interviews; $Q$, Questionnaire; $D^{*}$, Non-additive genetic effect is significant as evaluated by means of the chi-square difference test $(p<0.05)$; Age*, age difference in women is significant as evaluated by means of the chi-square difference test $(p<0.05)$

$\dagger$ Additive and non-additive genetic variance (ADE-model)

$¥$ The female-to-male ratio as evaluated by the logistic regression model and computation of odds-ratio

${ }^{1}$ Present study; ${ }^{2}$ Larsson et al. [8]; ${ }^{3}$ Svensson et al. [12]; ${ }^{4}$ Svensson et al. [13] 
be non-affected and decreased the prevalence from 23 to $16 \%$. A change of phenotype definition that lowers the prevalence but does not alter the heritability may reflect an overclassification of disease. The presence of non-genuine cases of migraine, such as migraine with aura without headache, infrequent migraine and secondary migraine is one possible explanation for this finding. Indeed, an increased familial risk was less apparent for secondary than for primary migraine [25]. False positive cases of migraine may also represent misclassification of tension-type headache (or another primary headache) and reporting error. Second, when cases of selfascertained migraine with other recurrent headache was rather considered to be non-affected and decreased the prevalence from 23 to $17 \%$, heritability dropped significantly (from 52 to $38 \%$ ). A change of phenotype definition that lowers the prevalence and the heritability is likely to reflect an underclassification of the trait. The presence of atypical cases is one plausible explanation for this finding. In accordance to the IHS classification, migrainous disorder is defined when all but one of the criteria for migraine is fulfilled. In one study of the Danish general population, about $10 \%$ of all migraine was diagnosed by experts as migrainous disorder [20]. Russell and Olesen concluded on the basis of family relative risk that most of these atypical cases were genuine cases of migraine [26]. Results from a separate validation study (published elsewhere) indicate that many of our false negatives of migraine did not fulfil the duration criterion due to reporting error. One may speculate whether genuine cases of migraine with infrequent relapses or long remission periods also are prone to reporting error. Noteworthy, the diagnosis of primary headache in one third of patients attending headache centres did not fulfil strict IHS criteria [27]. Thus, due to methodological reasons the proportion of migraine cases with atypical features may be as large as 25-50\% [28-32]. One major conclusion from this study is that the effect size of genetic influences on migraine may vary with the definition, wherein particular genetic information is lost due to underdiagnosis. To avoid the problem of ascertainment bias in epidemiological surveys of migraine it is important to account for atypical cases and reduce the effects of reporting error. Our results indicate that application of broad diagnostic criteria is one way to reach these aims.

The female-to-male ratio for the prevalence of our strict definition of migraine was almost 4 . In the broader definitions, female preponderance was lower. The genetic contribution of strict migraine tended to be greater for women than men ( $46 \%$ vs. $33 \%)$ but this difference was lower for the broad definition that accounted for presumably false negative cases ( $53 \%$ vs. $48 \%$ ). So, the problem of underdiagnosis seems to be somewhat larger in men than women. One possible explanation for sex-dependent ascertainment bias is that men are less severely affected by the disease. Our data strengthen the view of sex-limited scalar effects in migraine, where constitutional factors play a crucial role for female hypersensitivity.

An optimal definition of the phenotype is an important issue in aetiological studies of disease. Application of strict diagnostic guidelines in epidemiological designs may require some modification to avoid exclusion of genuine cases $[33,34]$. On more theoretical grounds case definition is expanded to include atypical cases or cases representing a sub-clinical stage of disease although they do not fully manifest disease according to strict diagnostic criteria [35]. Phenotypic sub-types of disease are coalesced into one broad diagnostic entity because they share some major aetiologic mechanism, which may be differently expressed in different individuals [36]. A more detailed anamnesis is required for exclusion of secondary cases, where causal factors are known and non-representative for primary disease, and classification into sub-types where phenotypic varieties have distinct liability distributions [9, 10]. Stratification of cases is applied when, for instance, a graded severity of disease reflects varying thresholds on the same single distribution of liability. Spontaneous and familial cases may represent a bimodal liability distribution. Several other stratification variables may be of aetiologic relevance for specific disease manifestation (age, gender, co-morbidity etc.). One approach to eliminate measurement error and obtain a more accurate definition of the phenotype is to perform longitudinal assessment [37]. In the present twin study, a relationship between definition and heritability of migraine was demonstrated and phenotypes of clinical and aetiological relevance delineated using cross-sectional analyses of multiple measures of the phenotype.

Acknowledgements Supported in part by a stipend from Karolinska Institutet to Dan A. Svensson. The Swedish Twin Registry is supported by grants from the Department of Higher Education, the Swedish Scientific Council and AstraZeneca. The SALT study was supported by grants from NIH (AG08724 to M. Gatz) and the Swedish Council for the Planning and Coordination of Research. The SALT Headache study was supported by grants from Merck Sharp \& Dohme.

\section{References}

1. Russell MB, Iselius L, Olesen J (1995) Inheritance of migraine investigated by complex segregation analysis. Hum Genet 96:726-730
2. Kors E, Haan J, Ferrari M (2003) Migraine genetics. Curr Pain Headache Rep 7:212-217
3. Harvald B, Hauge M (1956) A catamnestic investigation of Danish twins. Danish Med Bull 3:150-158 
4. Lucas RN (1977) Migraine in twins. J Psychosom Res 20:147-156

5. Gervil M, Ulrich V, Kyvik KO, Olesen J, Russell MB (1999) Migraine without aura: a population-based twin study. Ann Neurol 46:606-611

6. Ulrich V, Gervil M, Kyvik KO, Olesen J, Russell MB (1999) Evidence of a genetic factor in migraine with aura: a population-based Danish twin study. Ann Neurol 45:242-246

7. Honkasalo ML, Kaprio J, Winter T, Heikkilä K, Sillanpää M, Koskenvuo M (1995) Migraine and concomitant symptoms among 8167 adult twin pairs. Headache 35:70-78

8. Larsson B, Bille B, Pedersen NL (1995) Genetic influences in headaches: a Swedish twin study. Headache 35:513-519

9. Ulrich V, Gervil M, Kyvik KO, Olesen J, Russell MB (1999) The inheritance of migraine with aura estimated by means of structural equation modelling. J Med Genet 36:225-227

10. Gervil M, Ulrich V, Kaprio J, Olesen J, Russell MB (1999) The relative role of genetic and environmental factors in migraine without aura. Neurology 53:995-999

11. Ziegler DK, Hur YM, Bouchard TJ, Hassanein RS, Barter R (1998) Migraine in twins raised together and apart. Headache 38:417-422

12. Svensson DA, Larsson B, Waldenlind E, Pedersen NL (2003) Shared rearing environment in migraine: results from twins reared apart and twins reared together. Headache 43:235-244

13. Svensson DA, Larsson B, Waldenlind E, Pedersen NL (2002) Genetic and environmental influences on expression of recurrent headaches as a function of the reporting age in twins. Twin Res 5:277-286

14. Svensson DA, Larsson B, Bille B, Lichtenstein P (1999) Genetic and environmental influences on recurrent headaches in eight to nine-year-old twins. Cephalalgia 19:866-872

15. Stewart WF, Simon D, Shechter A, Lipton RB (1995) Population variation in migraine prevalence: a meta-analysis. J Clin Epidemiol 48:269-280
16. Rasmussen BK, Jensen R, Olesen J (1991) Questionnaire versus clinical interview in the diagnosis of headache. Headache 31:290-295

17. Tzourio C, Gagnière B, El Amrani M, Bousser M-G, Alpérovitch A (2003) Lay versus expert interviewers for the diagnosis of migraine in a large sample of elderly people. J Neurol Neurosurg Psychiatr 74:238-241

18. Pedersen NL, Lichtenstein P, Svedberg P (2002) The Swedish Twin Registry in the Third Millennium. Twin Res 5:427-432

19. - (1988) Classification and diagnostic criteria for headache disorders, cranial neuralgias and facial pain. Headache Classification Committee of the International Headache Society. Cephalalgia 8[Suppl 7]:1-96

20. Russell MB, Rasmussen BK, Thorvaldsen P, Olesen J (1995) Prevalence and sex ratio of the subtypes of migraine. Int J Epidemiol 24:612-618

21. Gervil M, Ulrich V, Olesen J, Russell MB (1998) Screening for migraine in the general population: validation of a simple questionnaire. Cephalalgia 18:342-348

22. Neale MC, Cardon LR (1992) Methodology for genetic studies of twins and families. Kluwer, London

23. Neale MC (1999) Mx: statistical modeling, 5th Edn. Department of Psychiatry, Richmond, VA

24. Joreskog KG, Sorbom D (1989) PRELIS 2. User's reference guide, 1st Edn. Scientific Software, Mooresville, USA

25. Russell MB, Olesen J (1996) Migraine associated with head trauma. Eur J Neurol 3:424-428

26. Russell MB, Olesen J (1996) Migrainous disorder and its relation to migraine without aura and migraine with aura. A genetic epidemiological study. Cephalalgia 16:431-435

27. Gallai V, Sarchielli P, Alberti A et al (2002) Application of the 1988 International Headache Society criteria in nine Italian headache centers using a computerized structured record. Headache 42:1016-1024
28. Henry P, Michel P, Brochet B, Dartigues JF, Tison S, Salamon R (1992) A nationwide survey of migraine in France: prevalence and clinical features in adults. GRIM. Cephalalgia 12:229-237

29. Göbel H, Petersen-Braun M, Soyka D (1994) The epidemiology of headache in Germany: a nationwide survey of a representative sample on the basis of the headache classification of the International Headache Society. Cephalalgia 14:97-106

30. Hagen K, Zwart J-A, Vatten L, Stovner LJ, Bovim G (2000) Prevalence of migraine and non-migrainous headache - head-HUNT, a large populationbased study. Cephalalgia 20:900-906

31. Dahlöf C, Linde M (2001) One-year prevalence of migraine in Sweden: a population-based study in adults. Cephalalgia 21:664-671

32. Henry P, Auray JP, Gaudin AF et al (2002) Prevalence and clinical characteristics of migraine in France. Neurology 59:232-237

33. Kendler KS, Neale MC, Kessler RC, Heath AC, Eaves LJ (1992) A population based twin study of major depression in women: the impact of varying definitions of illness. Arch Gen Psychiatry 49:257-266

34. Kendler KS, Pedersen NL, Neale MC, Mathé AA (1995) A pilot Swedish twin study of affective illness including hospital- and population-ascertained sub samples: results of model fitting. Behav Genet 25:217-232

35. Straub RE, MacLean CJ, O'Neill FA et al (1995) A potential vulnerability locus for schizophrenia on chromosome 6p24-22: evidence for genetic heterogeneity. Nat Genet 11:287-293

36. Gretarsdottir S, Sveinbjörnsdottir S, Jonsson HH et al (2002) Localization of a susceptibility gene for common forms of stroke to $5 q 12$. Am J Hum Genet 70:593-603

37. Kendler KS, Neale MC, Kessler RC, Heath AC, Eaves LJ (1993) The lifetime history of major depression in women: reliability of diagnosis and heritability. Arch Gen Psychiatry 50:863-870 